\title{
Pseudomyogenic Hemangioendothelioma of Bone Initially Managed as Slipped Capital Femoral Epiphysis: A Case Report
}

\author{
Nicole A. Friel ${ }^{1}$, Adam C. Rothenberg1 ${ }^{1}$, Kurt Weiss ${ }^{1,2}$ \\ ${ }^{1}$ Department of Orthopaedic Surgery, University of Pittsburgh Medical Center, Pittsburgh, USA \\ ${ }^{2}$ University of Pittsburgh Cancer Institute, University of Pittsburgh School of Medicine, Pittsburgh, USA \\ Email: weiskr@upmc.edu
}

Received 21 February 2014; revised 16 March 2014; accepted 23 March 2014

Copyright (C) 2014 by authors and Scientific Research Publishing Inc.

This work is licensed under the Creative Commons Attribution International License (CC BY). http://creativecommons.org/licenses/by/4.0/

(c) (i) Open Access

\begin{abstract}
Pseudomyogenic hemangioendothelioma is a rare, recently described neoplasm that usually presents as multifocal lesions in a single extremity. The disease has demonstrated a high propensity for infiltrative growth and local recurrence but limited metastatic potential. Variations of histological appearance and immunohistochemical signatures have been described, but typically involve spindle or polygonal cells with nuclear atypia and neutrophilic infiltration. Here we present a case report of an 8-year-old female who presented with hip pain that was initially diagnosed and managed as a slipped capital femoral epiphysis (SCFE). Subsequent evaluation led to the diagnosis of pseudomyogenic hemangioendothelioma of bone. Due to the degree of osseous destruction, described patterns of local recurrence, and metastatic potential of this neoplasm, a wide resection with endoprosthetic reconstruction of the proximal femur was performed. This case highlights the importance of due diligence in the diagnoses of SCFE and bone tumors in young patients with abnormalities of the proximal femur, including consideration of the need for biopsy.
\end{abstract}

\section{Keywords}

Pseudomyogenic Hemangioendothelioma, Slipped Capital Femoral Epiphysis, Sarcoma, Epithelioid

\section{Introduction}

Pseudomyogenic hemangioendothelioma is a recently described neoplasm that most often presents as multifocal lesions in one extremity. Over the last several years, additional descriptions of this disease have emerged, with 
characterization of its clinical, ultrastructural, and histological components [1]-[9]. This disease is poorly understood, diagnosis is challenging, and optimal management is uncertain. This rare tumor features locally aggressive behavior and high probability of recurrence but low metastatic potential. In this report, we describe a unique case of pseudomyogenic hemangioendothelioma that was first diagnosed as a slipped capital femoral epiphysis (SCFE) and only later appropriately diagnosed and managed.

\section{Case Presentation}

The patient is an eight year six month female who presented to a tertiary pediatric orthopaedic practice with a chief complaint of right hip pain for approximately one month after sustaining a fall. Prior to the fall she had been previously healthy and reported no antecedent right hip pain or any other medical problems. She was diagnosed with a slipped capital femoral epiphysis (SCFE) (Figure 1) and was admitted to the hospital for percutaneous screw fixation, which proceeded without complication. During her post-operative period, she was compliant with non-weight bearing instructions and her pain subsided. However, subsequent radiographs at 2 months post-operative showed infiltrative lucency, osseous destruction, and irregularity of the femoral head and neck (Figure 2). Endocrinologic and hematologic evaluations were negative. An infectious evaluation was performed and found to be negative. MRI and CT evaluation were non-diagnostic (Figure 3 and Figure 4), and she underwent CT-guided biopsy that was likewise inconclusive.

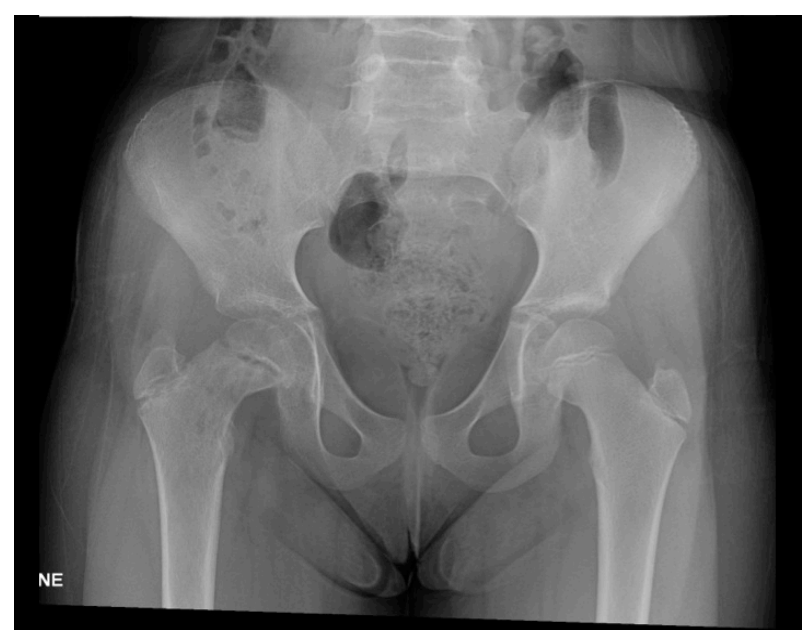

Figure 1. AP radiograph of the patient's pelvis at the time of her initial presentation. Her right capital femoral epiphysis is certainly widened, but the femoral neck and head have an abnormal, infiltrative appearance compared with the left side.

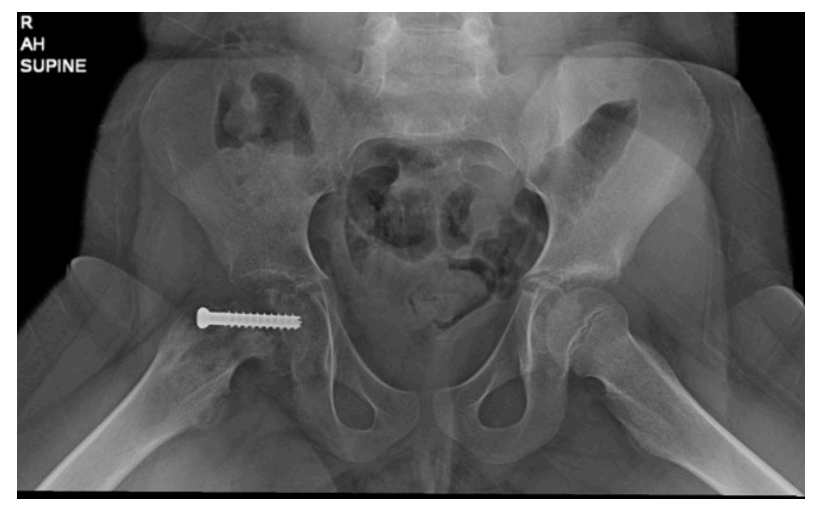

Figure 2. Shows the patient's frog-lateral radiograph subsequent to screw fixation. The bone is clearly abnormal on this image. 


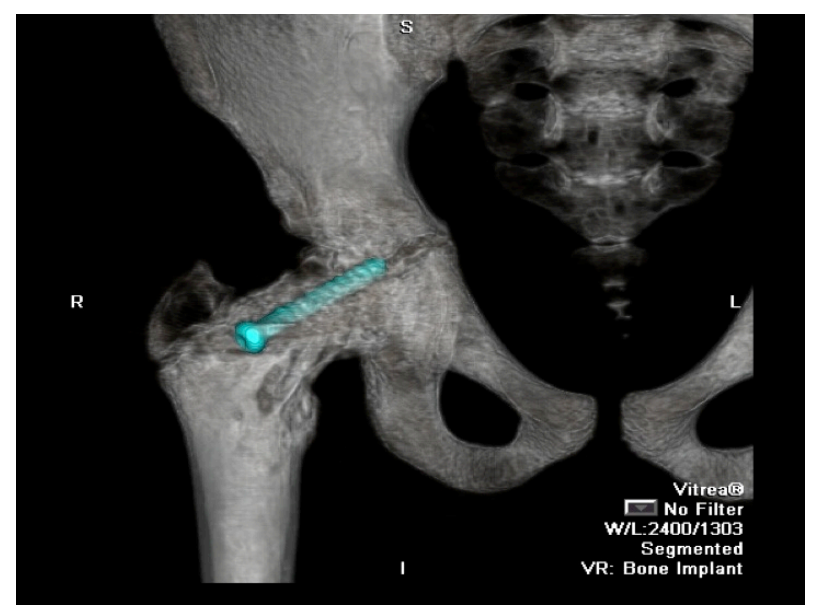

Figure 3. 3-D CT scan of the patients right hip suggesting an infiltrative process in the femoral neck and head.

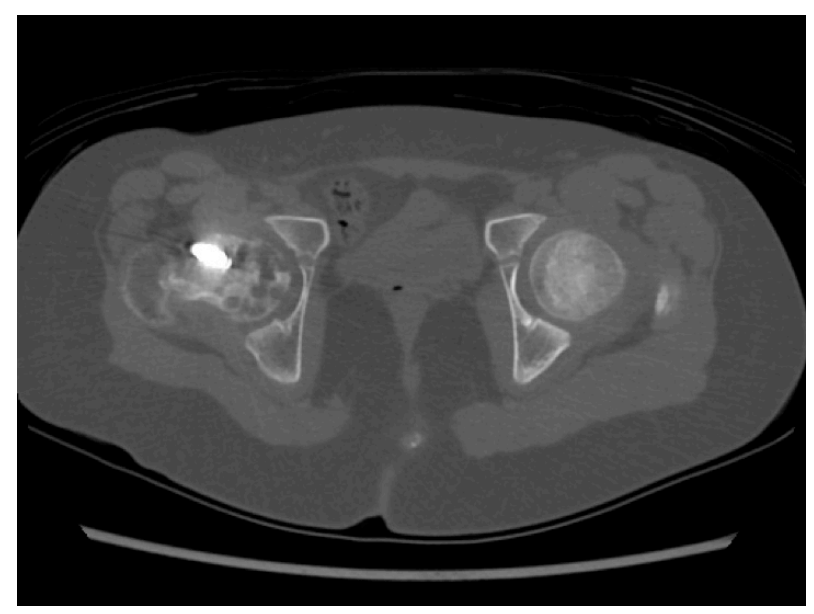

Figure 4. Displays an axial CT scan image, which demonstrates a lobular, infiltrative, lucent pattern within the femoral neck and head. The proximal femur was ultimately determined to be un-salvageable.

She was then referred to a musculoskeletal oncology service. Due to the non-diagnostic nature of her prior evaluations, she was taken to the operating room for an open biopsy through a lateral approach. Histologic evaluation showed foci of single and clustered epithelioid cells embedded within a fibrous stroma in the intertrabecular spaces. Reticulin stain demonstrated that most clustered cells were within vascular-type spaces. The cells immunostained variably for CD31 and cytokeratin AE1/AE3, with faint immunopositivity for smooth muscle actin (Figure 5 and Figure 6).

She was taken to the operating room and a wide resection (Figure 7) and endoprosthetic reconstruction were performed with negative histologic margins (Figure 8). There were no complications, and the patient progressed her weight bearing over several months post-operatively. The patient is currently 9 months post-operative and fully weight-bearing without pain or complaints. There is no clinical or radiographic evidence of recurrent disease.

\section{Discussion}

Pseudomyogenic hemangioendothelioma is rare, recently recognized disease entity. While the terminology "pseudomyogenic hemangioendothelioma" has only lately been established, multiple case series have reported on a constellation of clinical, morphological, and histological findings that likely describe similar disease entities 


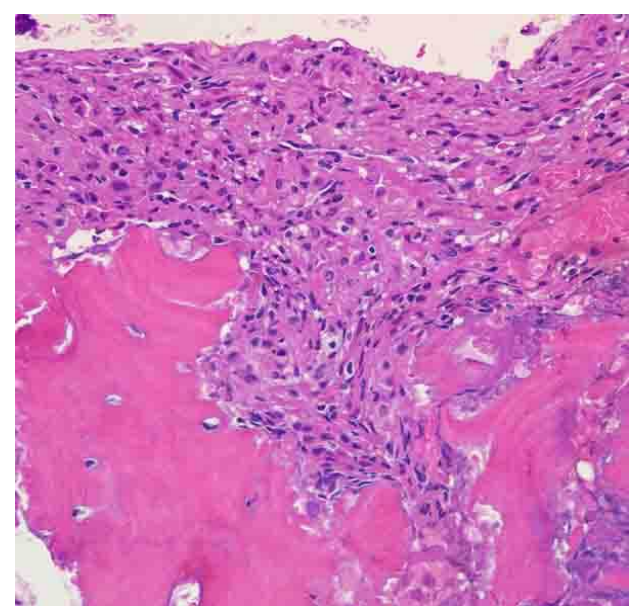

Figure 5. Shows a histological slide of the resected tumor (H \& E). Note the plump epithelioid-like cells growing in fascicles and invading the bone.
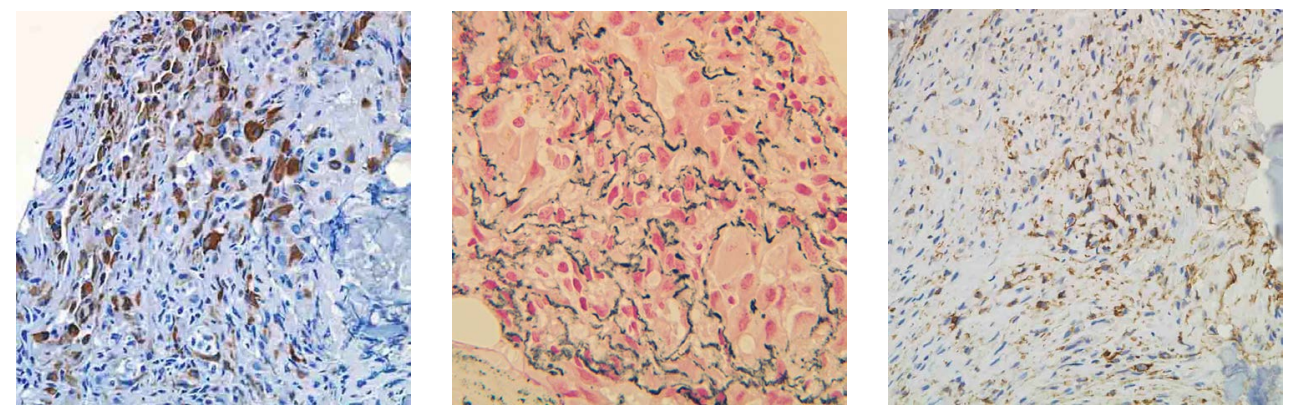

Figure 6. Immunohistochemistry pathology slides demonstrating (left) variable cytokeratin AE1/ AE3 staining (middle) reticulin stain demonstrating cell clustering within vascular-type spaces (right) variable CD31 staining.

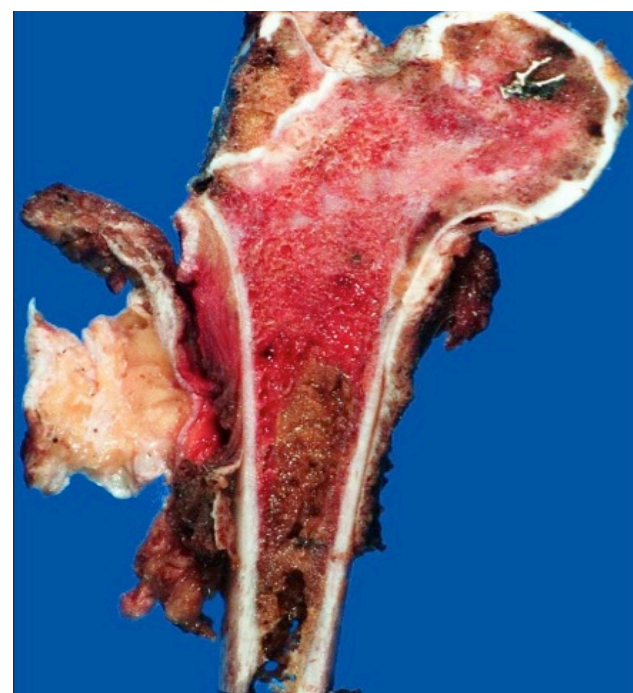

Figure 7. Presents the patient's bisected gross specimen. The lobular, abnormal tumor bone (pink) is in sharp contradistinction to the normal bone (brown). 


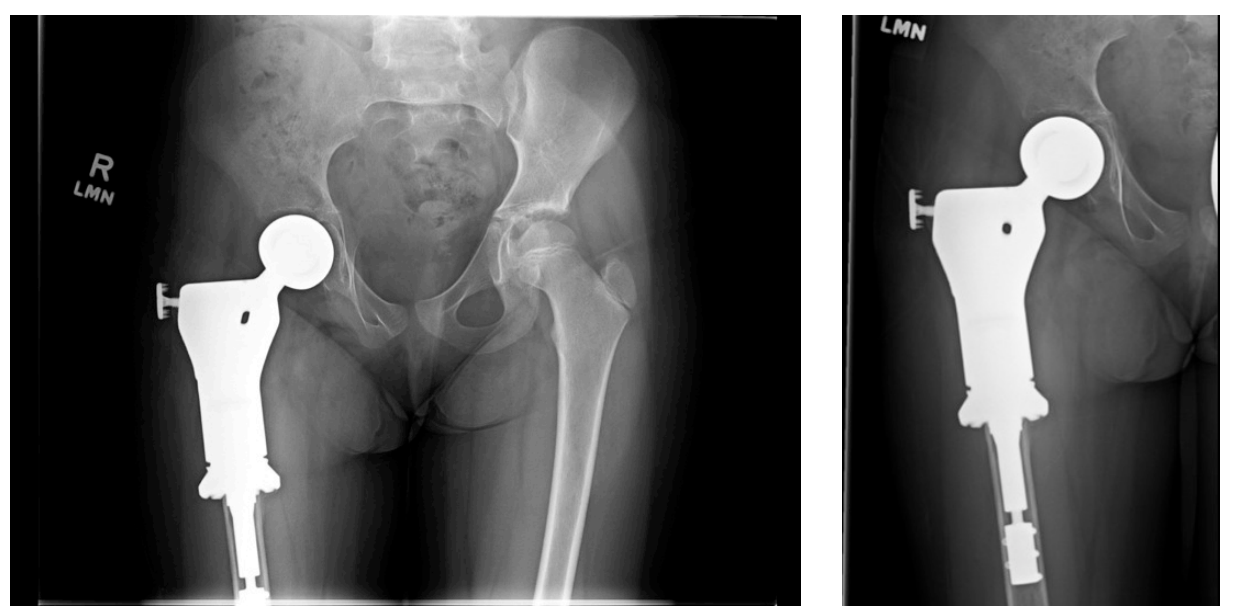

Figure 8. Display the patient's post-operative (left) AP pelvis and (right) AP hip views, respectively. A compressive osseointegration prosthesis was chosen to maximize the advantages of wide resection and biologic fixation.

[1]-[3]. Despite the multiple names associated with this disease process, it is referred to as pseudomyogenic hemangioendothelioma in the most recent WHO classification [4].

In 1992, Mirra et al. [3] described what the authors called a fibroma-like variant of epithelioid sarcoma in a small case series of 5 patients. The clinical presentation, ultrastructural features, and presence of vimentin and low molecular weight keratin within the tumor cells justified their designation as an epithelioid sarcoma variant. However, the tumors were unique morphologically and the authors categorized them separately. In 2003, Billings et al. [1] described seven tumors originally described as epitheliod sarcomas, but with distinctive morphologic and immunophenotypic features. Based upon further histologic examination, the cases were labeled epithelioid sarcoma-like hemangioendothelioma. In 2011, Hornick and Fletcher [2] described 50 cases of this distinctive tumor, describing its clinical presentation, behavior, morphology and immunophenotype. They named the tumor pseudomyogenic hemangioendothelioma. It is now generally proposed that these three papers describe the same rare neoplasm.

Microscopically, the neoplastic lesions are composed of plump spindle or polygonal cells, occasionally rhabdomyoblast-like, with abundant eosinophilic cytoplasm, and more rarely epithelioid cells arranged in ill-defined sheets, nodules or fascicles. Nuclear atypia is generally mild to moderate, but is occasionally prominent, and the mitotic rate is low with rare necrosis. Prominent infiltration by neutrophils is commonly seen. The lesions typically show a limited infiltrative growth pattern [5] [6]. Immunohistochemistry shows expression of cytokeratin AE1/AE3, FLI-1 and ERG, as well as expression of CD31 in half of all cases [1] [5]. Recently, a novel translocation, $\mathrm{t}(7 ; 19)$ (q22;q13), has been identified for pseudomyogenic hemangioendothelioma [9].

Pseudomyogenic hemangioendothelioma classically affects young adults, rarely over the age of 40, with a strong male predominance. Tumors usually involve the extremities and are multicentric, involving multiple tissues planes, from dermal, subcutaneous, subfascial, and importantly, osseous. Previous case series suggest an indolent course for this type of tumor [1] [2]. Tumors are treated with local excision/resection in most cases, even when there are multiple lesions. Local recurrence is common. Extensive multifocal disease in an extremity may necessitate amputation; the literature describes both transfemoral and transtibial amputations as well as a hip disarticulation. In review of the pseudomyogenic hemangioendothelioma literature, only three patients have developed distant metastases; one during the initial evaluation and the other two which occurred several years following excision/resection of the primary tumors [2] [3] [7].

Sheng and Wang [8] describe a unique case of pseudomyogenic hemangioendothelioma presenting as multifocal disease in the long bones of a 10-year-old girl. Lesions in her distal tibia and fibula as well as the distal femur were curetted and filled with bone cement; the distal femur lesion recurred with extension into the soft tissues. Further, this patient also developed a lesion of the proximal tibia several years later. The authors describe this multifocal disease as the first case of primary pseudomyogenic hemangioendothelioma of bone.

Pseudomyogenic hemangioendothelioma of bone is a rare tumor and the literature is not replete with guidance on how to optimally manage this disease. While the disease appears to have a limited metastatic potential, it has 
a high propensity for infiltrative growth and local recurrence. Given the local aggression and seemingly large area of disease in the proximal femur in the patient described in this case report, wide resection with endoprosthetic reconstruction was performed to minimize local recurrence. Especially due to the patient's young age, implant choice was challenging, but ultimately a proximal femoral replacement with compressive osseointegration was chosen to allow for biologic ingrowth while minimizing bony resection.

This case also highlights the importance of due diligence in the diagnosis of SCFE in a young patient with osteolytic abnormalities of the proximal femur as well as close radiographic follow-up in the post-operative period following screw fixation. An appropriate work-up was obtained to investigate her abnormality further and a broad differential diagnosis was entertained. Ultimately, the histological specimen was diagnostic. Further, although the first CT-guided biopsy was inconclusive, high clinical suspicion led to an open biopsy of the lesion, which led to the definitive diagnosis and treatment. Clearly, additional clinical and basic scientific research is required before a consensus on the appropriate treatment of this rare tumor is rendered.

\section{Acknowledgements}

The Authors wish to thank Dr. Ronald Jaffe of the Childrens Hospital of Pittsburgh Department of Pathology for his guidance and assistance.

This work was supported by NIH grant 1K08CA177927-01, the Shadyside Hospital Foundation, Pittsburgh CureSarcoma, the Pittsburgh Foundation, and the Houy family in loving memory of Jonathan Houy.

\section{References}

[1] Billings, S.D., Folpe, A.L. and Weiss, S.W. (2003) Epithelioid Sarcoma-Like Hemangioendothelioma. The American Journal of Surgical Pathology, 27, 48-57. http://dx.doi.org/10.1097/00000478-200301000-00006

[2] Hornick, J.L. and Fletcher, C.D. (2011) Pseudomyogenic Hemangioendothelioma: A Distinctive, Often Multicentric Tumor with Indolent Behavior. The American Journal of Surgical Pathology, 35, 190-201. http://dx.doi.org/10.1097/PAS.0b013e3181ff0901

[3] Mirra, J.M., Kessler, S., Bhuta, S. and Eckardt, J. (1992) The Fibroma-Like Variant of Epithelioid Sarcoma. A Fibrohistiocytic/Myoid Cell Lesion Often Confused with Benign and Malignant Spindle Cell Tumors. Cancer, 69, 1382-1395. http://dx.doi.org/10.1002/1097-0142(19920315)69:6<1382::AID-CNCR2820690614>3.0.CO;2-Y

[4] Jo, V.Y. and Fletcher, C.D. (2014) WHO Classification of Soft Tissue Tumours: An Update Based on the 2013 (4th) Edition. Pathology, 46, 95-104. http://dx.doi.org/10.1097/PAT.0000000000000050

[5] Cacciatore, M. and Dei Tos, A.P. (2014) Challenging Epithelioid Mesenchymal Neoplasms: Mimics and Traps. Pathology, 46, 126-134. http://dx.doi.org/10.1097/PAT.0000000000000063

[6] Mangham, D.C. and Kindblom, L.G. (2014) Rarely Metastasizing Soft Tissue Tumours. Histopathology, 64, 88-100. http://dx.doi.org/10.1111/his.12310

[7] Sheng, W., Pan, Y. and Wang, J. (2013) Pseudomyogenic Hemangioendothelioma: Report of an Additional Case with Aggressive Clinical Course. The American Journal of Dermatopathology, 35, 597-600. http://dx.doi.org/10.1097/DAD.0b013e31827c8051

[8] Sheng, W.Q. and Wang, J. (2012) Primary Pseudomyogenic Haemangioendothelioma of Bone. Histopathology, 61, 1219-1224. http://dx.doi.org/10.1111/j.1365-2559.2012.04347.x

[9] Trombetta, D., Magnusson, L., von Steyern, F.V., Hornick, J.L., Fletcher, C.D. and Mertens, F. (2011) Translocation t(7;19) (q22;q13)-A Recurrent Chromosome Aberration in Pseudomyogenic Hemangioendothelioma? Cancer Genetics, 204, 211-215. http://dx.doi.org/10.1016/j.cancergen.2011.01.002 